\title{
An Application of Multi-Attribute Value Theory to Patient-Bed Assignment in Hospital Admission Management: an Empirical Study
}

\author{
Pei-Fang (Jennifer) Tsai ${ }^{1 *}$ and Fu-Man Lin ${ }^{2}$ \\ ${ }^{1}$ Department of Industrial Engineering and Management, National Taipei University \\ of Technology, Taiwan, \\ ${ }^{2}$ Medical Affairs Department, Mackay Memorial Hospital, Taipei 10449, Taiwan
}

Submitted February 2014. Accepted for publication September 2014.

\begin{abstract}
Hospital beds are considered economically scarce and hospitalists strive to balance between utilizing beds more efficiently and complying with preference of physicians and patients when pairing patients to beds. This research is to develop preference-based decision rules for patient-bed assignment in a dynamic environment. A multi-attribute value theory (MAVT) model with additive value function is proposed to quantitatively deploy hospital policies in bed management. To elicit scaling factors and value functions for attributes, a linear programming model is constructed for all preference conditions. An empirical study was conducted with real data collected from two branches of a medical center. The simulated results using value function showed greater benefits when the patient-bed ratio was high and more flexible ward assignment was allowed. Further, a detailed analysis showed that this MAVT model was better in preference matching for both physicians/nurses and patients. At least 79 percent of patients were given beds in designated wards in accordance with their attending physicians' subspecialty, and more than 48 percent of patients' room preferences were matched in the simulated assignment for one branch.
\end{abstract}

Keywords: Preference-based decision, multi-attribute value function, waiting list prioritization, healthcare admission process

\section{INTRODUCTION}

Patient admission and inpatient bed assignment policy can have sophisticated influence on resource utilization for any hospital. With an effective admission process, a hospital can increase its turnover rate, reduce unnecessary bed occupancy, and improve the quality of care. Kumar et al. [1] studied the relationship between organizational structures and the performance of patient scheduling tasks. Their simulation results showed that different strategies are adopted in different organizational structures and increased centralization of information lends better performance due to more opportunities for planning and better information accuracy. In a study at Johns Hopkins Bayview Medical Center, a new active

*Corresponding author: Pei-Fang Jennifer Tsai, Ph.D., National Taipei University of Technology, No.1, Sec. 3, Zhongxiao E. Rd., Taipei 106 Taiwan. Tel: +886 (2) 2771-2171 ext 2354. Fax: +886 (2) 2731-7168. E-mail: ptsai@ntut.edu.tw. Other author: fmlin@ms1.mmh.org.tw. 
bed management scene that requires frequent assessment by hospitalists in bed availability was proposed. The implementation results showed significant improvement in the emergency department throughput and the availability of ICU beds [2].

In 1995, Taiwan government instituted a universal health insurance, the National Health Insurance (NHI), to make coverage available to everyone and the benefits have been significant since then [3]. Since more than 90 percent of Taiwan's health care providers are contracted with the NHI, it gives insurers the ultimate freedom in accessing medical services without any referral required. They can seek care directly at secondary or tertiary care institutions, regardless of the nature or severity of their illness. As a result, patients often go shopping for a doctor and are keen to express dismay on the quality of care. With few exceptions of certain costly high-tech treatments, there are effectively no ceilings on usage in the NHI. This has resulted in more stress on medical centers, high health care use rates, and a constant threat for the Bureau of National Health Insurance (BNHI) to restrain the growth in costs to make the universal insurance system financially sustainable [4]. To coerce hospitals and physicians to utilize resources more effectively, the BNHI has been experimenting designs of reimbursement system so that it would encourage hospitals to seek for welfare equilibrium among the degree of extension in either the patients' length of stay (day-based system, Length of Stay), number of services for patients (fee-for-servicebased system, Relative Value Units), and admission number of patients (per-case system, Diagnosis Related Groups) [5].

Statistical data from the BNHI showed that from 2002 to 2012, the inpatient payment has been about $33 \%$ of the total medical benefit claims on average while the budget has been steadily increased at a rate of $4 \%$ [6]. As shown in Figure 1, the average

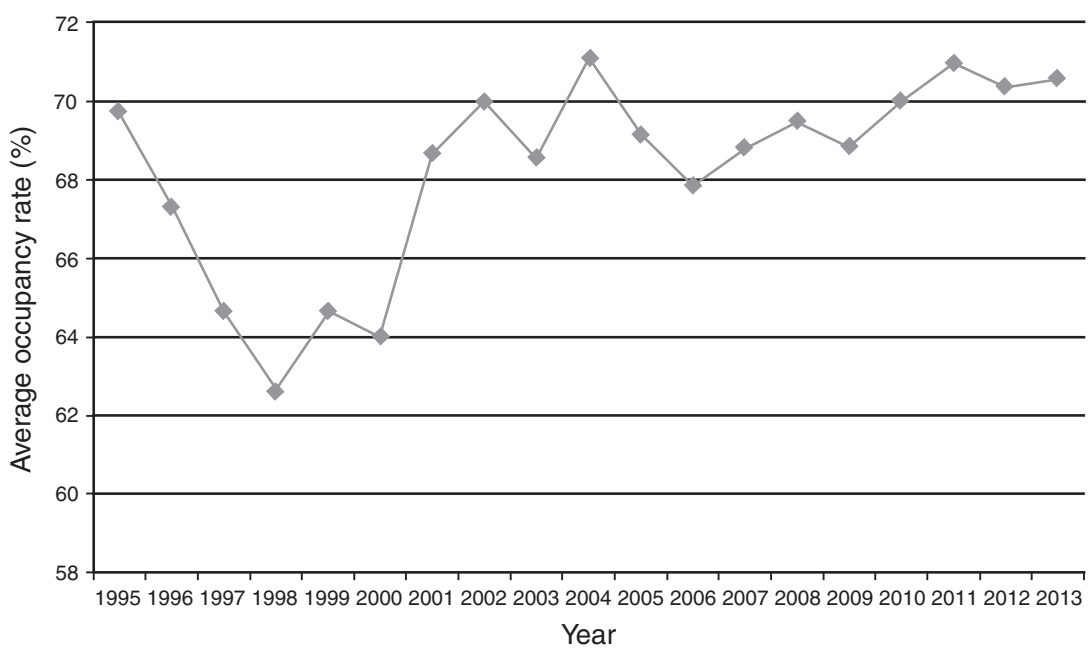

Figure 1. Average occupancy rates (\%) of general beds for Taiwan's hospitals from 1995 to 2013 [7]. 
occupancy rate including non-acute general beds has been ranged between $67 \%$ and $72 \%$ since 2001 [7]. High bed occupancy rate has been more pronounced in the academic medical centers in Taiwan due to higher demands. In 2011,79\% of academic medical centers had occupancy rates for acute general beds higher than $81.34 \%$, which was 1.2 times higher than the average of $67.78 \%$ for all hospitals [8].

When the bed supply is limited, one strategy for bed management is to increase occupancy rates. Compared with either reducing the average length of stay or the number of admissions, this option has the fewest consequences in the service level and competitiveness for hospitals. This might be achieved by reducing the share of beds kept empty for emergency cases, maintenance, and cleaning, or by more efficient planning [9]. An admission process begins with the generation of admission requests and ends with the actual admission of patients. Admissions can be categorized as either for emergency, scheduled, or elective patients. Compared to emergency admissions, bed managers have a better control over scheduled and elective admissions. Depending on their severity levels, some elective patients would need to be scheduled only on a specific day required by their physicians as others could be put on a waiting list. As the bed supply is restricted, the hospital has become a site of conflict where accessing a bed is becoming more problematic, and hence, a new position of bed manager has been institutionalized. The bed manager's role is central to hospital organizations as it provides a greater visibility in finding solutions to overcome the conflicting demands of planned procedures and the unpredictability of emergency admissions [10].

Previous studies mostly focused on the holistic view on the patients' admission scheduling and its impact on the utilization of multiple hospital resources. Hancock and Walter [11] proposed an "Admission Scheduling and Control System Simulator" to evaluate impacts of different admission policies on the workloads of hospital resources through simulating patient flows. A literature survey by Smith-Daniels et al. [12] concluded that most admission scheduling systems considered only the management of bed capacity, which caused sub-optimal use of other resources such as operating rooms. Harper and Shahani [13] focused on the hospital bed capacity plan based on the simulation study in bed occupancies and refused admission rates. Krusters and Groot [14] and Walczak et al. [15] proposed a decision support system framework for admission planning based on several prediction models on resource availability. Roth and van Dierdonck [16] considered the patients' service requirements and developed a Hospital Resource Planning (HRP) system, which adopted a master plan for capacity and material, and used the Diagnosis Related Groups (DRG) system for patient classification. Based on demand forecast, the HRP system reserved the capacity for urgent and emergency patients in the master plan. Gemmel and van Dierdonck [17] summarized the system requirements for admission processes as (1) an admission policy for emergencies, urgencies, and elective patients; (2) expected capacity utilization among hospital resources; (3) projection of patients' requirements for services and resources; and (4) the organizational structure for a workable scheduling system.

While the primary issue for hospitals in admission management is to anticipate the uncertainty of the arrival of emergency patients, there would be more leeway for hospitalists to manage the waiting list strategically. Worthington [18] simulated the impact 
of alternative scenarios in managing waiting lists by individual consultant. Mullen [19] gave a literature review on waiting list management. To represent the dynamic of waiting lists, Gorunescu et al. [20] used queueing theory to study the effect of changing admission rates, length of stay and bed assignment on bed occupancy, emptiness and rejection in departments of geriatric medicine. Goddard and Tavakoli [21] used queueing models to analyze the welfare and efficiency gains in various policies regarding accessing and prioritizing the waiting list. They suggested policy of implicit rationing in waiting list accessibility, which was to manage the waiting list by illness-severity-related clinical priority to determine the speed of patients to move through the list. Vissers et al. [22] used simulation to compare four admission concepts in scheduling both emergency and elective patients, and concluded that, when the level of resource occupancy was high, reserving capacity for emergency patients was shown to be effective.

It seems natural for hospitals to adopt information systems since processes in health care services heavily rely on information. However, challenges still exist in building information systems to fully support various health care processes [25]. Haux [26] found that most hospitals still maintained paper-based systems even after introducing computer-based systems. The research argued that development in future health information systems needs to be directly correlated with better efficiency of care to ensure the care is affordable. Tsai et al. [27] made similar observation in one of Taiwan's general hospitals which considered information systems only for data storage rather than an integral part of the actual processes, even in administrative processes. The reluctance in paradigm shifts might be due to the gap between the system design and the users' expectation on system usability.

This research focuses on the decision problem in patient-bed assignment at a patient placement center. Instead of formulating the patient-bed assignment problem as a scheduling problem as in Demeester et al. [23] and Schmidt et al. [24], this research considers a dynamic approach with preference-based decision rules. As in reality, the waiting list is constantly changing throughout the day and the decision in patient-bed assignments would be continuously made whenever a hospital bed becomes available. Hence, this study focuses on how to quantify preference, or value, to rank patients on the waiting list according to the characteristics of each available bed. The eligible patients from the waiting list would be ranked based on the value of the available bed.

The remainder of the paper is organized as follows. In the next section, the process to develop our multiple attribute value theory (MAVT) model and the mathematical formulation used as the elicitation technique are depicted. The implementation results based on the empirical study and the detail analysis are then included in sections 3 and 4 , respectively. Finally, concluding remarks and research limitations are summarized in section 5 .

\section{THE MAVT MODEL}

This study was approved by the National Taipei University of Technology Institutional Review Board for protection of human subjects in research. For most hospitals, the priority in patient selection needs to comply with the general policies and be consistent through all admissions. Similar to medical preference problem, the admission staff's 
Table 1. List of major factors to determine patients' priority in the selected hospital

\begin{tabular}{|c|c|c|}
\hline \multicolumn{3}{|c|}{ Surgical Specialty } \\
\hline Importance & Factor & Preference \\
\hline High & Treatment type & Surgery $>$ Chemotherapy $>$ Others \\
\hline & Ward subspecialty & Designated subspecialty $>$ Supported subspecialty \\
\hline$\downarrow$ & Days in waiting & More days passed scheduled date \\
\hline Low & Patient's room preference & $\begin{array}{l}\text { Matched in single, double room, or insurance- } \\
\text { covered room }\end{array}$ \\
\hline \multicolumn{3}{|c|}{ Internal Medicine Specialty } \\
\hline Importance & Factor & Preference \\
\hline High & Treatment type & $\begin{array}{l}\text { Advanced laboratory testing }>\text { Chemotherapy }> \\
\text { Others }\end{array}$ \\
\hline \multirow[t]{2}{*}{$\downarrow$} & Ward subspecialty & Designated subspecialty $>$ Supported subspecialty \\
\hline & Days in waiting & More days passed scheduled date \\
\hline Low & Patient's room preference & $\begin{array}{l}\text { Matched in single, double room, or insurance- } \\
\text { covered room }\end{array}$ \\
\hline
\end{tabular}

prioritization on patients can be described as a problem that searches for the best utilization of resources while preference is addressed in ordinal comparisons of attributes among alternatives for a specific resource [28, 29]. The major factors and the importance of these factors toward the patient prioritization for hospital bed in surgical and internal medicine specialties in our case study are summarized in Table 1 . The notion $\alpha \succ \beta$ represents that the decision strictly prefers $\alpha$ to $\beta$. For the surgical specialty, the highest priority is given to those patients who are scheduled for surgery on a specific date. Internal medicine patients who are hospitalized for cardiovascular related disease or scheduled kidney biopsy are given higher priority to secure the accessibility of advanced laboratory testing.

The goal of this patient admission process is to find the right patients for the available beds at the right time. To meet this goal, an admission center might be focusing on two fundamental objectives: to maximize the utilization of medical resources and to maximize patients' satisfaction. The first two factors listed in Table 1, treatment type and ward subspecialty, are related to the use of medical resources. Due to the limited availability of operating rooms and advanced testing equipment such as Positron Emission Tomography (PET), the consequences of not admitting scheduled patients on the requested dates can be costly. Similar to the ward subspecialty, aggregating patients according to their medical needs in the same ward is beneficial logistically for attending physicians and managerial for the ward nurses to care their patients. For patients' satisfaction, the admission staff strives to admit patients as soon as possible (fewer days in waiting) with the consideration of their room preference (single, double, or insurance-covered room). 
Table 2. Definition of attributes and levels used to prioritize patients in empirical case

\begin{tabular}{llc}
\hline Factor (Attribute) & \multicolumn{1}{c}{ Description } & Attribute level \\
\hline Treatment type $\left(x_{1}\right)$ & Surgery or advanced laboratory testing & $x_{1}=2$ \\
& Chemotherapy & $x_{1}=1$ \\
& Others & $x_{1}=0$ \\
Ward subspecialty $\left(x_{2}\right)$ & Designated subspecialty & $x_{2}=1$ \\
& Supported subspecialty & $x_{2}=0$ \\
Days in waiting $\left(x_{3}\right)$ & Days passed scheduled date (no more & $x_{3}=$ passed days (0 9) \\
& than 9 days) & $x_{4}=1$ \\
Room preference $\left(x_{4}\right)$ & Available bed matches the request & $x_{4}=0$ \\
& Otherwise & \\
\hline
\end{tabular}

Table 2 summarizes the list of attributes and associated attribute levels used in this case study. These attributes are considered proxy attributes, which serve as indirect measures to the fundamental, not easily quantifiable objective in how to best admit patients [32]. Each attribute has several levels where a more preferred situation is assigned a higher attribute level. For example, a surgery patient (with attribute level of 2 , or $x_{1}=2$ ) would be preferred over a chemotherapy patient (with attribute level of 1 , or $\left.x_{1}=1\right)$ according to the guideline in Table 1 .

For multi-attribute value theory (MAVT), the formulation of value functions can be additive or multiplicative. Under the von Neumann-Morgenstern rationality axioms, a weighted value function is used to rank alternatives based on the consequences and trade-off between attributes [30,31]. There are two assumptions to be verified for applicability of MAVT: preferential independency and weak-difference independency [32]. Since the decision of patient-bed assignment is made by a limited number of admission staff and the guideline in Table 1 is strictly followed, the assumption of preferential independency and weak-difference independency is then considered appropriate in this case. Given a set of single attribute value functions, $u_{i}\left(x_{i}\right), i=1, \ldots, n$, and a multi-attribute value function $u\left(x_{1}, \ldots, x_{\mathrm{n}}\right)$, an additive value function is used if there exists a set of scaling factors, $k_{i}, i=1, \ldots, n$, such that $u\left(x_{1}, \ldots, x_{n}\right)=\sum_{i=1}^{n} k_{i} u_{i}\left(x_{i}\right)$ and $\sum_{i=1}^{n} k_{i}=1$ [32]. To construct an additive value function, it is to determine both the value function for each attribute as well as the associated scaling factor individually [30]. However, the procedure to elicit parameters in the value function often depends on the assumption of specific shapes in value/utility functions or is user-dependent [33]. This study adopts the elicitation procedure proposed by Wu and Gonzalez $(1996,1998)$ for a weighting function regardless of the form of the value function [34]. Moreover, this is a special case of that procedure since the probability weightings are reduced to constant scales. Suppose $a_{1}$ and $a_{2}$ are two alternatives with associated attribute levels $x_{i}\left(a_{1}\right)$ and $x_{i}\left(a_{2}\right), i=1,2$, respectively. The prospect theory (PT) [35] can be reduced to the following: 


$$
\begin{aligned}
& \left(k_{1}, x_{1}\left(a_{1}\right) ; k_{2}, x_{2}\left(a_{1}\right)\right) \succ\left(k_{1}, x_{1}\left(a_{2}\right) ; k_{2}, x_{2}\left(a_{2}\right)\right) \\
& \Leftrightarrow k_{1} x_{1}\left(a_{1}\right)+k_{2} x_{2}\left(a_{1}\right)>k_{1} x_{1}\left(a_{2}\right)+k_{2} x_{2}\left(a_{2}\right)
\end{aligned}
$$

where $0 \leq k_{1} \leq 1,0 \leq k_{2} \leq 1$, and $k_{1}+k_{2} \leq 1$.

After multiple consensus meetings with admission staff and managers, six general preference conditions for different decision scenarios are derived. Let $u(a)=u\left(x_{1}(a)\right.$, $\left.x_{2}(a), x_{3}(a), x_{4}(a)\right)$ be the value of some patient $a$ with levels of $x_{1}(a), x_{2}(a), x_{3}(a)$, and $x_{4}(a)$, for attributes $x_{1}, x_{2}, x_{3}$, and $x_{4}$, respectively. Moreover, let $x_{i}(a)=*$ represent an arbitrary level for attribute $x_{i}, i=1, \ldots, 4$. The preference conditions can be summarized as follows:

Preference condition 1: Patients scheduled for surgery or advanced laboratory testing have the highest priority over chemotherapy patients and other patients; that is, $u(2, *, *, *)>u(1, *, *, *)$ and $u(2, *, *, *)>u(0, *, *, *)$.

Preference condition 2: For chemotherapy patients, those whose attending physicians belong to one of the dedicated subspecialties of the available beds get priority; that is, $u(1,1, *, *)>u(1,0, *, *)$.

Preference condition 3: When the conditions in days of waiting and the room preference are identical, a non-chemotherapy patient whose attending physician belongs to one of the dedicated subspecialties of the available beds has higher priority; that is, $u(0,1, t, 1)>u(1,0, t, 1)$ and $u(0,1, t, 0)>u(1,0, t, 0)$.

Preference condition 4: When the conditions in days of waiting are identical, chemotherapy patients whose room preferences are matched have higher priority over other patients whose room preferences are not matched even if their attending physicians belong to one of the dedicated subspecialties of the available beds; that is, $u(1,0, t, 1)>u(0,1, t, 0)$.

Preference condition 5: When the conditions in the room preference are identical, chemotherapy patients who waited for one day more have the same priority as the other patients whose attending physicians belong to one of the dedicated subspecialties of the available beds; that is, $u(1,0, t+1,1)=u(0,1, t, 1)$ and $u(1,0, t+$ $1,0)=u(0,1, t, 0)$.

Preference condition 6: When the attending physician does not belong to one of the dedicated subspecialties of the available beds, a chemotherapy patient has priority over the patients in the "Others" category; that is, $u(1,0, *, *)>u(0,0, *, *)$.

To determine the value for each attribute level as well as the scaling factor, we first define variables $\tilde{u}_{i}\left(x_{i}\right)=k_{i} \cdot u_{i}\left(x_{i}\right)$ as a weighted value function for attribute $x_{i}, i=1, \ldots, 4$. Moreover, the condition of having arbitrary level for attribute $x_{i}$ is assumed to be bounded by $\max \left\{\tilde{u}_{i}\right\}$, the maximum weighted value for attribute $x_{i}, i=$ $1, \ldots, 4$. Then we formulate and solve an equivalent mathematical programming problem defined as follows:

Minimize $\quad z=\sum_{j=0}^{2} \tilde{u}_{1}(j)+\sum_{j=0}^{1} \tilde{u}_{2}(j)+\sum_{j=0}^{9} \tilde{u}_{3}(j)+\sum_{j=0}^{1} \tilde{u}_{4}(j)$

Subject to

$$
\tilde{u}_{1}(2) \geq \tilde{u}_{1}(1)+\max \left\{\tilde{u}_{2}\right\}+\max \left\{\tilde{u}_{3}\right\}+\max \left\{\tilde{u}_{4}\right\}+\varepsilon,
$$




$$
\begin{gathered}
\tilde{u}_{1}(1)+\tilde{u}_{2}(1) \geq \tilde{u}_{1}(1)+\tilde{u}_{2}(0)+\max \left\{\tilde{u}_{3}\right\}+\max \left\{\tilde{u}_{4}\right\}+\varepsilon, \\
\tilde{u}_{1}(0)+\tilde{u}_{2}(1) \geq \tilde{u}_{1}(1)+\tilde{u}_{2}(0)+\varepsilon, \\
\tilde{u}_{1}(1)+\tilde{u}_{2}(0)+\tilde{u}_{4}(1) \geq \tilde{u}_{1}(0)+\tilde{u}_{2}(1)+\tilde{u}_{4}(0)+\varepsilon, \\
\tilde{u}_{1}(1)+\tilde{u}_{2}(0)+\tilde{u}_{3}(t+1)=\tilde{u}_{1}(0)+\tilde{u}_{2}(1)+\tilde{u}_{3}(t), \text { for } t \in\{0,1,2, . ., 8\} \\
\tilde{u}_{1}(1) \geq \tilde{u}_{1}(0)+\max \left\{\tilde{u}_{3}\right\}+\max \left\{\tilde{u}_{4}\right\}+\varepsilon, \\
\text { and } \tilde{u}_{i}(\cdot) \geq 0, i=1, \ldots, 4 .
\end{gathered}
$$

It is simply to find a feasible solution that satisfies all constraints in this mathematical model and an artificial objective function, as in Equation (1), is included to serve as a searching direction for an optimal solution. Equations (2) to (7) enforce the ordering as stated in preference conditions 1 to 6 , respectively. Further, a relaxation parameter $\varepsilon$ is used to impose the strict preference ranking in all conditions. Equation (8) ensures non-negative weighted values for all attributes at all levels.

Without loss of generality, the non-linear terms in this formulation can be replaced by linear terms, for example, to replace $\max \left\{\tilde{u}_{2}\right\}$ with $\tilde{u}_{2}(2)$, under the assumption that the weighted value functions should be non-decreasing along with increasing attribute levels. Let $\varepsilon=0.5$, and the resulting formulation can then be solved using a linear programming solver with the optimal solution of $\tilde{u}_{1}(0)=0, \tilde{u}_{1}(1)=6, \tilde{u}_{1}(2)=18.5$, $\tilde{u}_{2}(0)=0, \tilde{u}_{2}(1)=6.5, \tilde{u}_{3}(t)=0.5 t, t \in\{0,1,2, . .9\}, \tilde{u}_{4}(0)=0$, and $\tilde{u}_{4}(1)=1$, with the objective value $z=54.5$.

After obtaining the optimal solution, the values are then normalized. For the value function of each attribute, it can simply be calculated by $u_{i}\left(x_{i}\right)=\tilde{u}_{i}\left(x_{i}\right) / \max \left\{\tilde{u}_{i}\right\}$ such that the values at different levels are ranged from 0 to 1 . The largest weighted value occurs when all attributes are at their highest level, that is, $\max \{\tilde{u}\}=\tilde{u}_{1}(2)+\tilde{u}_{2}(1)+\tilde{u}_{3}(9)+\tilde{u}_{4}(1)=30.5$. Since the largest value for each attribute is assumed to be 1 , this summation is equivalent to the sum of scaling factors. Then the normalized scaling factor is defined as follows:

$$
k_{i}=\frac{\max \left\{\tilde{u}_{i}\right\}}{\max \{\tilde{u}\}}, i=1, \ldots, 4 .
$$

Table 3 summarizes the final value function and the scaling factor for each attribute in this basic additive multi-attribute value model. Suppose that a surgical patient needs a surgery with a predetermined admission date of today, and his/her attending physician belongs to one of the dedicated subspecialties of that available bed, this patient would get a value of 0.852 for that bed when the patient's room preference is met.

\section{RESULTS}

Our study hospital has two campuses in Taipei municipal area: Tamshui branch and Main hospital. With a total capacity of more than 1500 acute beds, these two campuses provide inpatient services for outpatient clinics from medical specialties such as 
Table 3. The value function and scaling factors for the additive value model in an empirical case

\begin{tabular}{lcc}
\hline Factor (Attribute) & Scaling factor & Value function \\
\hline Treatment type $\left(x_{1}\right)$ & $k_{1}=0.607$ & $u_{1}(2)=1, u_{1}(1)=0.324, u_{1}(0)=0$ \\
Ward subspecialty $\left(x_{2}\right)$ & $k_{2}=0.213$ & $u_{2}(1)=1, u_{2}(0)=0$ \\
Days in waiting $\left(x_{3}\right)$ & $k_{3}=0.148$ & $u_{3}\left(x_{3}\right)=x_{3} / 9, x_{3}=0,1, \ldots, 9$. \\
Room preference $\left(x_{4}\right)$ & $k_{4}=0.032$ & $u_{4}(1)=1, u_{4}(0)=0$ \\
\hline
\end{tabular}

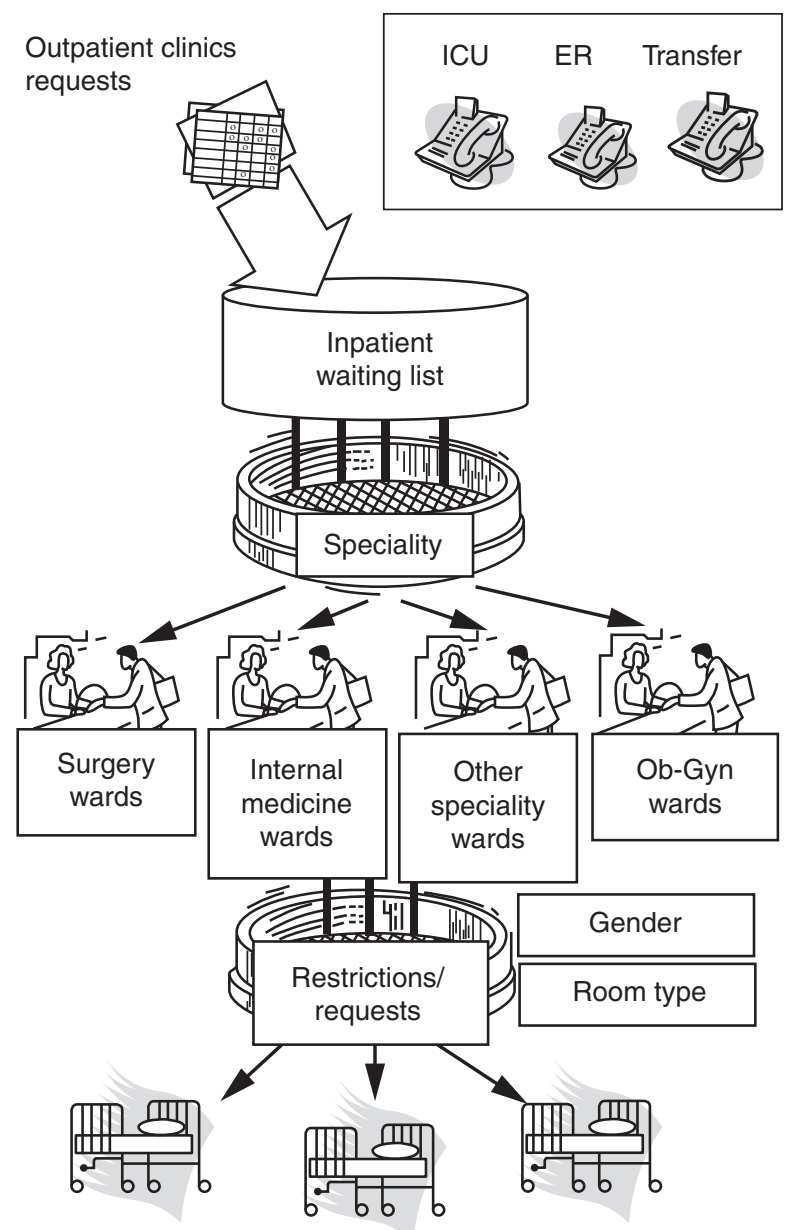

Figure 2. Centralized patient-bed assignment process in patient placement center.

internal medicine, surgery, pediatrics, obstetrics and gynecology. Other than outpatient clinics, transfer requests for admission might also come from emergency room (ER) and intensive care unit (ICU). Figure 2 shows the process in assigning patients to hospital beds in the patient placement center. 
The inpatient requests by outpatient clinics will enter a single waiting list and will be called in on or after the admission dates depending on the bed availability. Hospital beds are divided into wards and each ward supports more than one medical specialties based on the strategic planning of bed capacity in the hospital. The current admission policy is to first dynamically reserve certain available beds for ER and ICU at the beginning of each day. Whenever a bed is released and available for the next patient, the scheduler is informed to select the next patient from the waiting list. The waiting list is managed on a first-come first-served basis, but other key factors and constraints are also taken into consideration, such as specialty, treatment requirement, degree of urgency, gender, and even preference of room choice. In general, most patients are admitted within 3 days after their request. However, if delay in admission is critical, patients who fail to be assigned to beds need to have another appointment with their attending physicians for new admission dates. The maximum allowable delay on waiting list is set to be 9 days after the request by hospital managers for chronic disease patients, as shown in Table 2.

Our case study focuses on the requests from outpatient clinics, which account for two-third of the bed capacity for the entire hospital. The data were collected from both campuses of this academic medical center. Even though there is only one inpatient center in this hospital, the waiting lists for these two campuses are kept separately and bed sharing is seldom considered unless further requested by physicians. Moreover, the decisions of patient-bed assignment on two campuses are made by two designated teams of staff. To validate the effectiveness of the proposed value model as shown in Table 3, real data of patient-bed assignment were collected from the patient placement center on both campuses.

The experiment is to mimic the actual patient-bed assignment process, and hence a decision support system (DSS) is developed to incorporate the function for managing bed data, patient data, and patients-bed matching. When a decision maker finds an available inpatient bed, the system will automatically rank patients according to their values to the bed. Assumptions in simulating the patient-bed assignment process are as follows:

Assumption 1. At the beginning of each day, the entire list of patients who are eligible to be admitted as well as available hospital beds are given.

Assumption 2. Cancellations and postponements are excluded in the simulated data. If any request is cancelled, or the preferred admission date is postponed or changed, the patient will be removed from the waiting list.

Assumption 3. The same sequence is used in searching for available beds. The decision maker will move to the next department only after either all available beds are assigned, or no more eligible patient needs a bed.

Assumption 4. The decision maker always selects the patient with the highest value for an available bed. If more than one patient has the same highest value, choose randomly.

\subsection{Results for Tamshui Branch}

Tamshui branch is an 800-bed unit for both acute and non-acute cares. Once patients are required to be hospitalized, they need to submit the hospitalization requests to the admission center at least a day prior to the bed assignment, with or without a preferred 
Table 4. The 3-day admission summary for Tamshui branch

\begin{tabular}{|c|c|c|c|c|c|c|}
\hline & \multicolumn{4}{|c|}{ Number of elective patients } & \multirow{2}{*}{$\begin{array}{l}\text { Number of } \\
\text { available } \\
\text { beds }\end{array}$} & \multirow{2}{*}{ Patient- } \\
\hline & $\begin{array}{l}\text { With preferred } \\
\text { admission date }\end{array}$ & $\begin{array}{c}\text { No preferred } \\
\text { admission date }\end{array}$ & $\begin{array}{c}\text { Same-day } \\
\text { request }\end{array}$ & Total & & \\
\hline Day 1 & 3 & 11 & 22 & 36 & 34 & 1.06 \\
\hline Day 2 & 1 & 7 & 16 & 24 & 19 & 1.26 \\
\hline Day 3 & 5 & 5 & 24 & 34 & 27 & 1.26 \\
\hline
\end{tabular}

Table 5. Similarity measures of MAVT model for Tamshui branch (in \%)

\begin{tabular}{lccc}
\hline & Patient Sameness & Assignment Similarity & Assignment Sameness \\
\hline Day 1 & 94.1 & 94.1 & 26.5 \\
Day 2 & 94.7 & 94.7 & 52.6 \\
Day 3 & 88.9 & 85.2 & 33.3 \\
Average & 92.6 & 91.3 & 37.5 \\
\hline
\end{tabular}

admission date. It is also possible to have same-day requests from the outpatient clinics. Three days of admission data have been collected and the general information is summarized in Table 5. In Table 4, the patient-bed ratio is the ratio of the total number of patients on the waiting list to the total number of available beds. When patient-bed ratio is less than 1, it indicates the supply of beds is sufficient for inpatient demands. It was observed that the number of new admissions in this branch is tightly regulated by physicians according to the bed availability.

The effectiveness of value function is measured using three ratios: patient sameness, assignment similarity, and assignment sameness. Compared to the actual assignment, patient sameness represents the percentage of same patients that are selected by the MAVT model, and assignment sameness is the percentage of patients that are paired with the same bed. To demonstrate the effectiveness of the value model, we define a relaxed index of assignment sameness, referred to as assignment similarity that represents the percentage of patient-bed pairs in which patients are assigned to either the same beds, beds in the same wards, or beds in compatible wards as in the actual assignment.

The simulated results of ranking patients with the additive value function are summarized in Table 5. The results using the multi-attribute value function were remarkable with an average of 92.6 percent and 91.3 percent in patient sameness and assignment similarity, respectively. It indicated that the bed-patient assignments by the experts in the bed management team were able to follow the hospital's guideline very closely. It might be also because many wards in this branch were dedicated to only one subspecialty in this branch. On the other hand, a higher percentage in assignment sameness, such as 52.6 percent on day 2, suggested that the degree of freedom in these assignments might be limited. According to the admission staff, many available beds could fit only a fairly limited subgroup of patients as the bed assignments were under a tighter control among the doctors. 


\subsection{Results for Main Hospital}

The Main hospital is located in the city center of Taipei, with 900 acute care beds in total. Since more outpatient clinics for various subspecialties are provided, a higher volume of outpatient visits as well as longer waiting list of elective patients were observed. The actual admission data for an entire week, from Monday to Saturday, are summarized in Table 6. With a significant number of same-day requests from the outpatient clinics, the patient-bed ratio greatly fluctuated due to the variation of the number of available beds every day. A high bed turnover rate was observed to accommodate more patients on the waiting list. Moreover, except for Saturday (day 6), it was observed that the number of elective patients was relatively stable.

The simulated results are summarized in Table 7 . The results using the multiattribute value function had an average of 79.1 percent and 64.7 percent in patient sameness and assignment similarity, respectively. It indicated that the bed-patient assignments by the experts in this bed management team were trying to follow the hospital's guideline most of the time, but not as closely as Tamshui branch. Compared to Tamshui branch, the Main hospital had more elective patients with preferred admission dates for surgery and advanced lab testing, a larger number of admissions, and a longer waiting list per day. A much lower percentage in assignment sameness, 19 percent on average, indicated a much higher flexibility, and hence more complexity, in assigning beds to patients in this branch. Not only there were more patients with similar condition on the waiting list, but most wards were designated to multiple subspecialties.

Table 6. The 6-day admission summary for the Main hospital

\begin{tabular}{|c|c|c|c|c|c|c|}
\hline & \multicolumn{4}{|c|}{ Number of elective patients } & \multirow{2}{*}{$\begin{array}{c}\text { Number of } \\
\text { available } \\
\text { beds }\end{array}$} & \multirow[b]{2}{*}{$\begin{array}{l}\text { Patient- } \\
\text { bed ratio }\end{array}$} \\
\hline & $\begin{array}{l}\text { With preferred } \\
\text { admission date }\end{array}$ & $\begin{array}{c}\text { No preferred } \\
\text { admission date }\end{array}$ & $\begin{array}{c}\text { Same-day } \\
\text { request }\end{array}$ & Total & & \\
\hline Day 1 & 66 & 21 & 25 & 112 & 74 & 1.51 \\
\hline Day 2 & 47 & 39 & 19 & 105 & 54 & 1.94 \\
\hline Day 3 & 63 & 43 & 19 & 125 & 73 & 1.71 \\
\hline Day 4 & 48 & 44 & 11 & 103 & 52 & 1.98 \\
\hline Day 5 & 52 & 34 & 17 & 103 & 62 & 1.66 \\
\hline Day 6 & 10 & 31 & 20 & 61 & 36 & 1.69 \\
\hline
\end{tabular}

Table 7. Similarity measures of MAVT model for the Main hospital (\%)

\begin{tabular}{lccc}
\hline & Patient Sameness & Assignment Similarity & Assignment Sameness \\
\hline Day 1 & 83.8 & 70.3 & 13.5 \\
Day 2 & 79.6 & 70.4 & 18.5 \\
Day 3 & 78.1 & 61.6 & 11.0 \\
Day 4 & 78.8 & 59.6 & 23.1 \\
Day 5 & 82.3 & 59.7 & 14.5 \\
Day 6 & 72.2 & 66.7 & 33.3 \\
Average & 79.1 & 64.7 & 19.0 \\
\hline
\end{tabular}




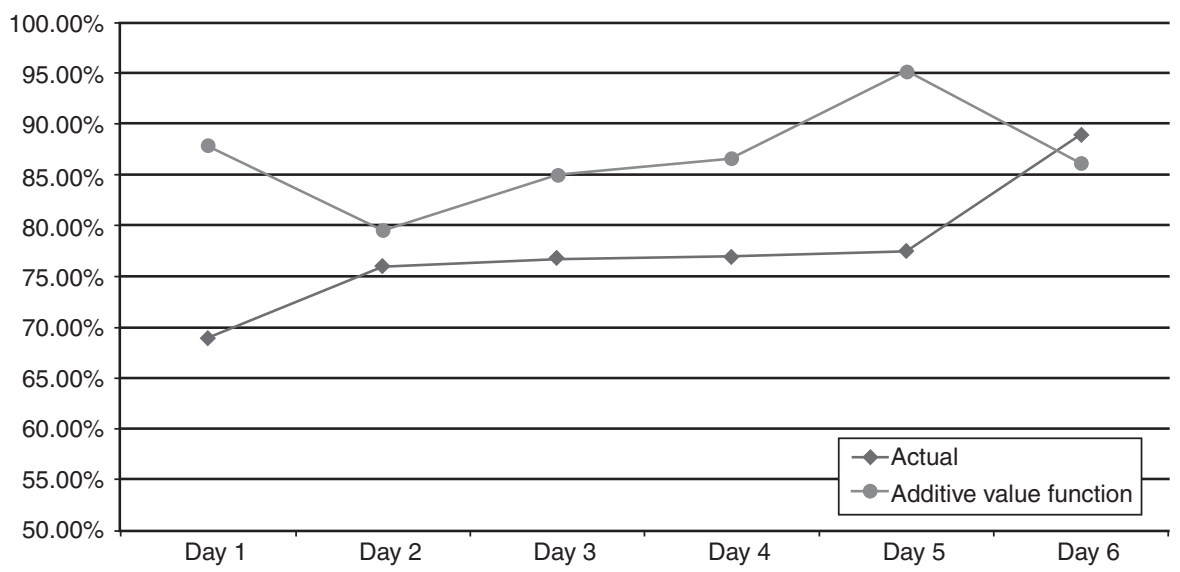

Figure 3. Percentage of compliance in patient-bed assignments to designated subspecialty wards.

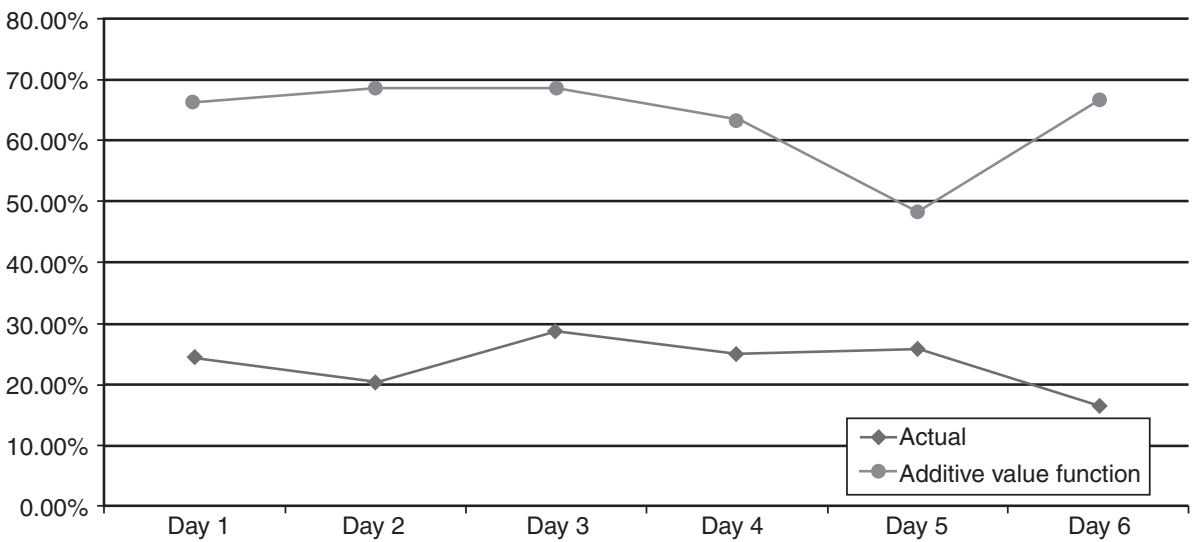

Figure 4. Percentage of compliance in patient-bed assignments to patients' room preferences.

Next, the quality of patient-bed assignments in terms of ward subspecialty compliance and room preference compliance was investigated. Figure 3 shows the percentage of patients assigned to designated wards in accordance with subspecialty of their attending physicians in the actual assignments and that by using additive value function. The Mann-Whitney test shows that the level of ward subspecialty compliance is significantly higher when using MAVT model with a corresponding $p$ value of 0.045 . At least 79 percent of the patients were assigned beds in the designated wards in the assignments using MAVT model, and the improvement in compliance could be up to 18 percent as in day 1 .

Figure 4 shows the percentage of patients assigned to rooms matched to their preferences. The level of room preference compliance is also significantly higher when 
using MAVT model with a corresponding $p$ value of 0.005 . More than 48 percent of the patients were assigned to rooms that fitted their preference in the assignments using MAVT model. On the contrary, no more than 30 percent of patients had their room preferences matched in the actual assignment. The improvement could be up to 50 percent as in day 6 .

\section{DISCUSSION}

To develop a preference-based decision for patient-bed assignment in a dynamic environment, this research proposes a MAVT model to quantitatively deploy hospital policies in the bed management. Once a consensus is reached among hospital managers and doctors on the priority rules among various possible scenarios, it is described as a set of preference rules. To elicit value and scaling parameters, a linear programming model is constructed to translate these preference rules into associated constraints with weighted attribute values. If an optimal solution is available, it is further converted by normalization to find the value functions for attributes as well as the scaling factors to construct the associated value model.

In this study, an additive value model was derived based on a set of six preference conditions. As shown in Table 3, not all value functions for attributes were linear. For example, Figure 5(a) shows the value function for attribute $x_{1}$, or patients' treatment type. At attribute level $x_{1}=1$, the value was 0.324 and was closer to the value for level $x_{1}=0$ than level $x_{1}=2$. It can be observed that, in general, chemotherapy patients were preferred over patients for other treatment, but this advantage did not dominate in preference condition 3. For attribute $x_{3}$, or the days in waiting, the value function was found to be linear as shown in Figure 5(b). When this factor was considered, as in preference condition 5 , it was only to decide whether a patient had been waited one-day longer than the other patient, without any specification on the number of days in waiting.

The scaling factors in this value model, as depicted in Figure 6, reflect how each attribute was weighted in the decision process. For attribute $x_{1}$, the scaling factor $k_{1}$ was greater than 0.5 and hence the priority in treatment type was predominant in the

(a)

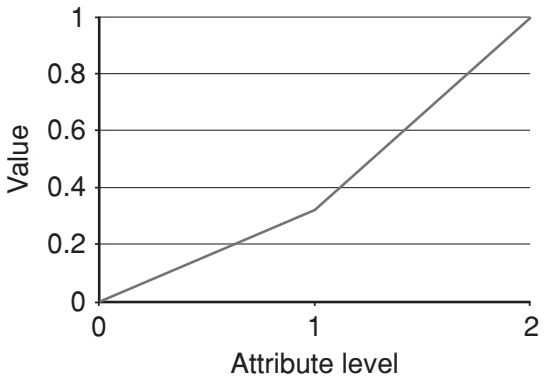

(b)

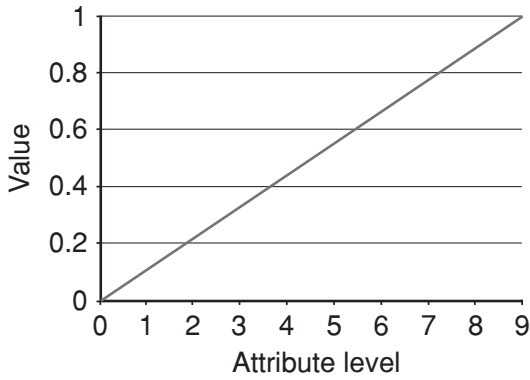

Figure 5. (a) The value function for treatment type. (b) The value function for days in waiting. 


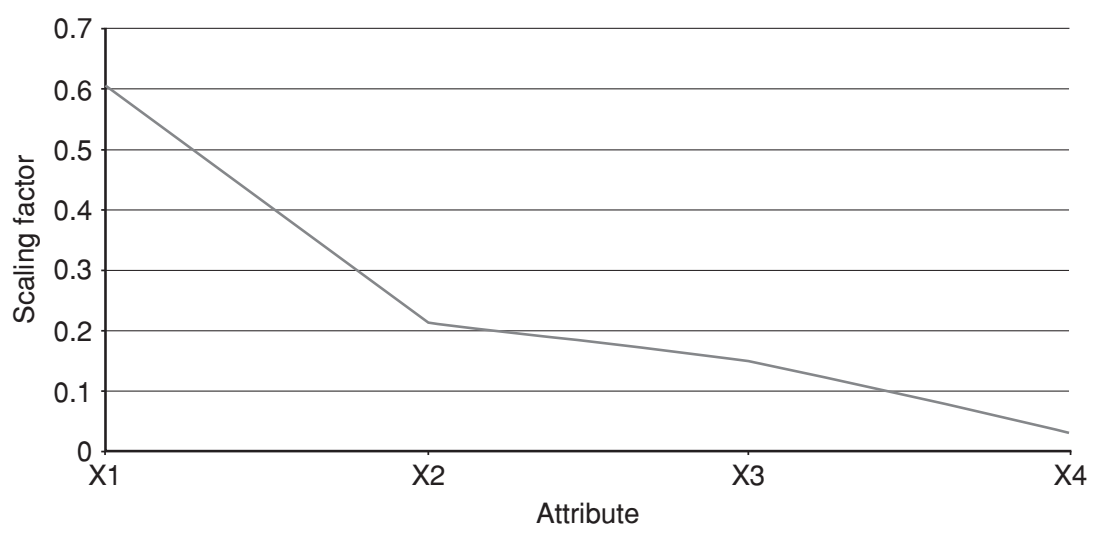

Figure 6. The scaling factors for all attributes.

preference ranking. As for attribute $x_{2}$ and $x_{3}$, the difference in their scaling factors was merely 0.065 , which was the smallest among all pairs of adjacent attributes in $\left\{x_{1}, x_{2}\right\}$ and $\left\{x_{3}, x_{4}\right\}$. This could be due to the indifference ranking as in preference condition 5 .

In the empirical study, it was found that when the waiting list is tightly regulated, as the case in Tamshui branch, the effectiveness of utility functions was less significant. The simulated results had an average of 92.6 percent in patient sameness and 91.3 percent in assignment similarity when using MAVT. In Tamshui branch, the admission requests were carefully managed based on doctors' rationale as the availability of beds was fully under their control. To synchronize the waiting list to the estimated bed availability, a daily meeting is mandated for all doctors in this branch. The admission summary in Table 4 showed that the patient-to-bed ratio is not high, and even close to 1 , suggesting that about one patient is waiting for each available bed on that day. Due to highly regulated demand, Tamshui branch also had a higher ratio of same-day admission requests than the Main hospital.

However, such arrangement might not be feasible for most general hospitals such as the Main hospital in Taipei. Its admission process was far more dynamic as outpatient clinics continued to send in hospitalization requests. It was more likely to have patients with severe conditions and the waiting list was constantly updated and re-evaluated during the day. For patients appointed to operating rooms and advanced examination equipment schedule, the consequence might be severe if the hospital failed to admit them on the preferred admission dates. Moreover, to accommodate rare inpatient requests from subspecialties such as breast surgery or dentistry, and also to increase the bed turnover rate for the entire hospital, the admission staff was given a higher flexibility in managing wards. Hence, the simulated results using additive value function amounted to a lower average of 79.1 percent and 64.7 percent in patient sameness and assignment similarity, respectively, and a much lower percentage in assignment sameness, 19 percent on average, in the Main hospital.

A lower level in patient sameness, assignment similarity, or assignment sameness implies a greater potential in using additive value model to ensure that the decisions in 
patient-bed assignments coincide with the consensus. This phenomenon was observed in the quality of the patient-bed arrangement for the Main hospital in Taipei. The assignment using MAVT model had a higher ward subspecialty compliance and room preference compliance, as shown in Figure 3 and Figure 4. The benefit of having high ward subspecialty compliance is three-fold. For attending physicians, the traveling distance and time needed to care for their patients in their daily routine could be reduced when all his/her patients were in the same set of wards. For ward nurses, it would confine the medical orders complexity and hence less error-prone. From the perspective of hospital management, high ward subspecialty compliance means less logistical concerns and better control in medical inventory. This additive value model was able to allocate more than 79 percent, even up to 95 percent, of patients to beds in designated wards in accordance with their attending physicians' subspecialty. The room preference compliance is important to patients' satisfaction in the healthcare service provided by the hospital. At least 48 percent, even up to 68 percent, of patients had their room preferences matched.

With the encouraging results, however, one major limitation of this study was the limited number of factors and attributes considered in the MAVT model. As observed in the admission center, when multiple patients are equally suitable for a bed, they might be further distinguished according to other minor situation-dependent factors that need to be further investigated.

\section{CONCLUSION}

Hospital bed management has become the central issue in the solutions to overcome the conflicting demands of planned procedures and the unpredictability of emergency admissions. Fierce competition among hospitals and the desire to better utilize the beds had made managing patients waiting list a stressful job. Lack of transparency and effectiveness in the decision of bed assignment has led to frustration and dissatisfaction of patients, hospital staff, and physicians. This research aims to provide a dynamic decision support system for hospital managers and physicians in the patients waiting list management.

In this research, a multi-attribute value theory (MAVT) model is proposed and a weighted value function is used to capture the essences in admission decisions. The proposed elicitation procedure for the MAVT model was to solve an equivalent mathematical programming model, which requires no assumption in the form and type of the value function. The current empirical study showed the advantages of this model applied to patient-bed assignment in real hospitals. Moreover, the quality of patient-bed assignment was greatly improved by using this additive value function in terms of ward subspecialty compliance and patients' room preference compliance.

\section{ACKNOWLEDGEMENTS}

The study has received financial support from the National Taipei University of Technology - Mackay Memorial Hospital Joint Research Program (NTUT-MMH-9913). The author would like to thank Po-Chia Chen, Hong-Jyun Chen, Hao-Yuan Sung, and the entire team of administrative staff from the participating hospital for assistance and support. Thanks are also due to the anonymous reviewers for their valuable comments and suggestions. 


\section{CONFLICT OF INTEREST}

The authors indicated no potential conflicts of interest.

\section{REFERENCES}

[1] Kumar A, Ow PS, Prietula, MJ. Organizational simulation and information systems design: An operations level example. Management Science, 1993, 39(2):218-239.

[2] Howell E, Bessman E, Kravet S, Kolodner K, Marshall R, Wright S. Active bed management by hospitalists and emergency department throughput. Annals of Internal Medicine, 2008, 149(11):804-810.

[3] Wen CP, Tsai SP, Chung WSI. A 10-year experience with universal health insurance in Taiwan. Annals of Internal Medicine, 2008, 148(4):258-267.

[4] Cheng TM. Taiwan's new national health insurance program: genesis and experience so far. Health Affair, 2003, 22(3): 61-76.

[5] Ministry of Health and Welfare, Bureau of National Health Insurance. TW-DRGs Improve Healthcare Quality, Efficiency and Fairness. http://www.mohw.gov.tw/EN/PDA/Detail.aspx?f_list_no=378\&fod_ list_no=4999\&doc_no=45308, Accessed September 29, 2014.

[6] Ministry of Health and Welfare. National Health Expenditure 2012. http://www.mohw.gov.tw/ EN/Ministry/Statistic.aspx?f_list_no=474 Accessed September 29, 2014.

[7] Ministry of Health and Welfare. Hospital and Clinic Statistics 2013: Table 11 Occupancy Rate of Hospital Beds, 1998-2013. http://www.mohw.gov.tw/EN/Ministry/Statistic.aspx?f_list_no=474 . Accessed September 29, 2014.

[8] Ministry of Health and Welfare, National Health Insurance Administration. Occupancy Rate of Hospital Acute Beds and Chronic Beds 2009-2011. http://www.nhi.gov.tw/Resource/webdata/20092011\%E5\%9B\%9B\%E9\%A1\%9E\%E7\%97\%85\%E5\%BA\%8A\%E5\%B9\%B4\%E5\%8D\%A0\%E5\% BA\%8A\%E7\%8E\%87\%E4\%B8\%80\%E8\%A6\%BD\%E8\%A1\%A8\%28101.08.14\%29.pdf. Accessed September 29, 2014.

[9] Kroneman M, Siegers JJ. The effect of hospital bed reduction on the use of beds: a comparative study of 10 European countries. Social Science \& Medicine, 2004, 59:1731-1740.

[10] Nairn S. The politics of beds. Accident and Emergency Nursing, 2003, 11:68-74.

[11] Hancock W, Walter P. The ASCS: inpatient admission scheduling and control system. Technical report, Ann Arbor, MI: AUPHA Press, 1983.

[12] Smith-Daniels VL, Schweikhart SB, Smith-Daniels DE. Capacity management in health care services: review and future research directions. Decision Science, 1988, 19(4):889-919.

[13] Harper PR, Shahani AK. Modelling for the planning and management of bed capacities in hospitals. Journal of the Operational Research Society, 2002, 53(1):11-18.

[14] Kusters RJ, Groot PMA. Modelling resources availability in general hospitals: design and implementation of a decision support model. European Journal of Operational Research, 1996, 88:428-445.

[15] Walczak S, Pofahl WE, Scorpio RJ. A decision support tool for allocating hospital bed resources and determining required acuity of care. Decision Support System, 2002, 43:445-456.

[16] Roth A, van Dierdonck R. Hospital resource planning: concepts, feasibility, and framework. Production and Operations Management, 1995, 4(1):2-29.

[17] Gemmel P, van Dierdonck R. Admission scheduling in acute care hospitals: does the practice fit with the theory? International Journal of Operations and Production Management, 1999, 19(9):863-878.

[18] Worthington D. Hospital waiting list management models. Journal of the Operational Research Society, 1991, 42(10): 833-843.

[19] Mullen PM. Waiting lists in the post-review NHS. Health Services Management Research, 1999, 7(2):131-145. 
[20] Gorunescu F, McClean SI, Millard PH. Using a queueing model to help plan bed allocation in a department of geriatric medicine. Health Care Management Science, 2002, 5:307-312.

[21] Goddard J, Tavakoli M. Efficiency and welfare implications of managed public sector hospital waiting lists. European Journal of Operational Research, 2008, 184:778-792.

[22] Vissers JMH, Adan IJBF, Dellaert NP. Developing a platform for comparison of hospital admission systems: an illustration. European Journal of Operational Research, 2007, 180 (3):1290-1301.

[23] Demeester P, Souffriau W, De Causmaecker P, Vanden Berghe G. A hybrid tabu search algorithm for automatically assigning patients to beds. Artificial Intelligence in Medicine, 2010, 48(1):61-70.

[24] Schmidt R, Geisler S, Spreckelsen C. Decision support for hospital bed management using adaptable individual length of stay estimations and shared resources. Medical Informatics and Decision Making, 2013, 13(3):19 pages.

[25] Lenz R, Reichert M. IT support for healthcare processes-premises, challenges, perspectives. Data \& Knowledge Engineering, 2007, 61:39-58.

[26] Haux R. Health information systems - past, present, future. International Journal of Medical Informatics, 2006, 75: 268-281.

[27] Tsai PF, Chen PC, Chiu CY, Chen KY. Information system evaluation for hospital admission management: a case study. In: Doolen T, van Aken E (Eds.), Proceedings of the 2011 Industrial Engineering Research Conference, Reno NV, May 2011.

[28] Krischer JP. Utility structure of a medical decision-making problem. Operations Research, 1976, 24(5):951-972.

[29] Torrance GW, Boyle MH, Horwood SP. Application of multi-attribute utility theory to measure social preferences for health states. Operations Research, 1982, 30(6):1043-1069.

[30] Fishburn PC. Independence in utility theory with whole product sets. Operations Research, 1965, 13(1):28-45.

[31] Keeney RL. Multiplicative utility functions. Operations Research, 1965, 22(1):22-34.

[32] Keeney RL, Raiffa H. Decisions with Multiple Objectives: Preferences and Value Tradeoffs, Cambridge, UK: Cambridge University Press 1993.

[33] Abdellaoui M. Parameter-free elicitation of utility and probability weighting functions. Management Science, 2000, 46(11):1497-1512.

[34] Wu G, Gonzalez R. Curvature of the probability weighting function. Management Science, 1996, 42(12):1676-1690.

[35] Tversky A, Fox CR. Weighting risk and uncertainty. Psychological Review, 1995, 102:269-283. 


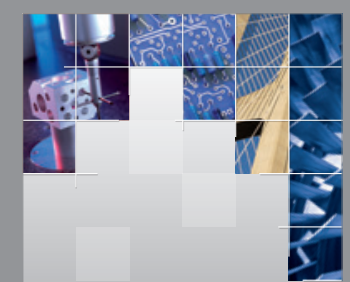

\section{Enfincering}
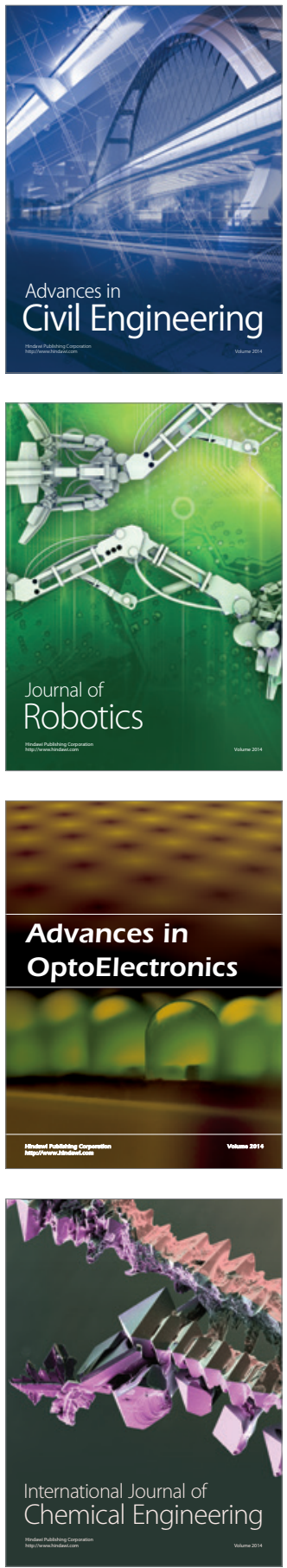

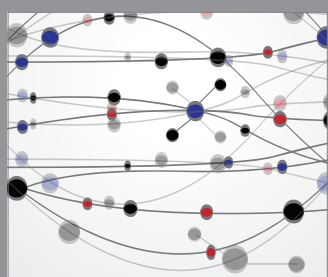

The Scientific World Journal

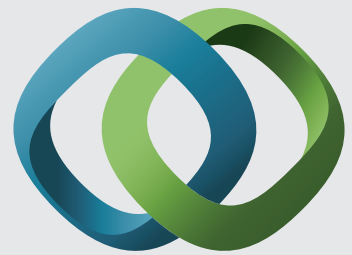

\section{Hindawi}

Submit your manuscripts at

http://www.hindawi.com
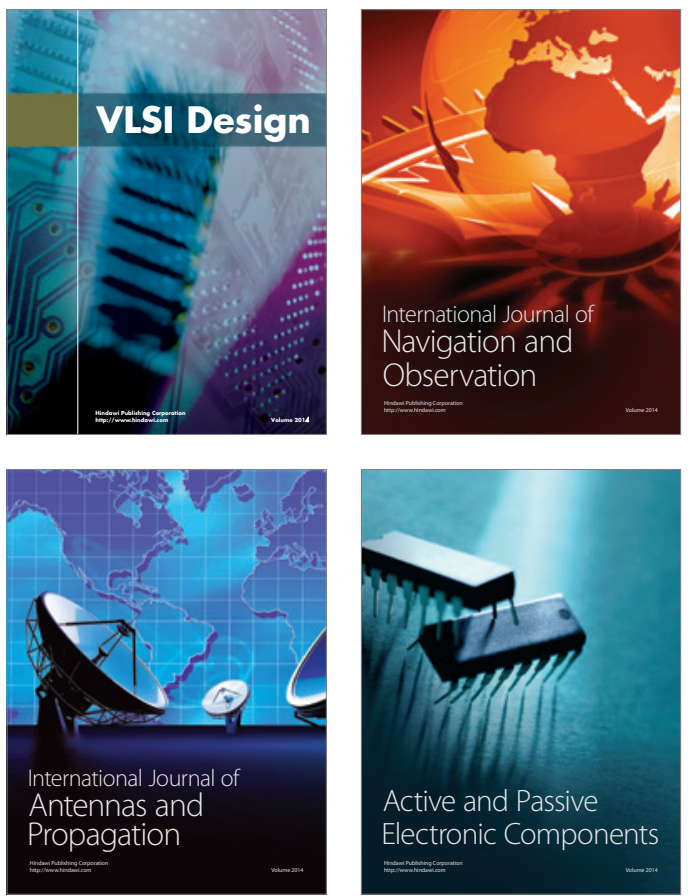
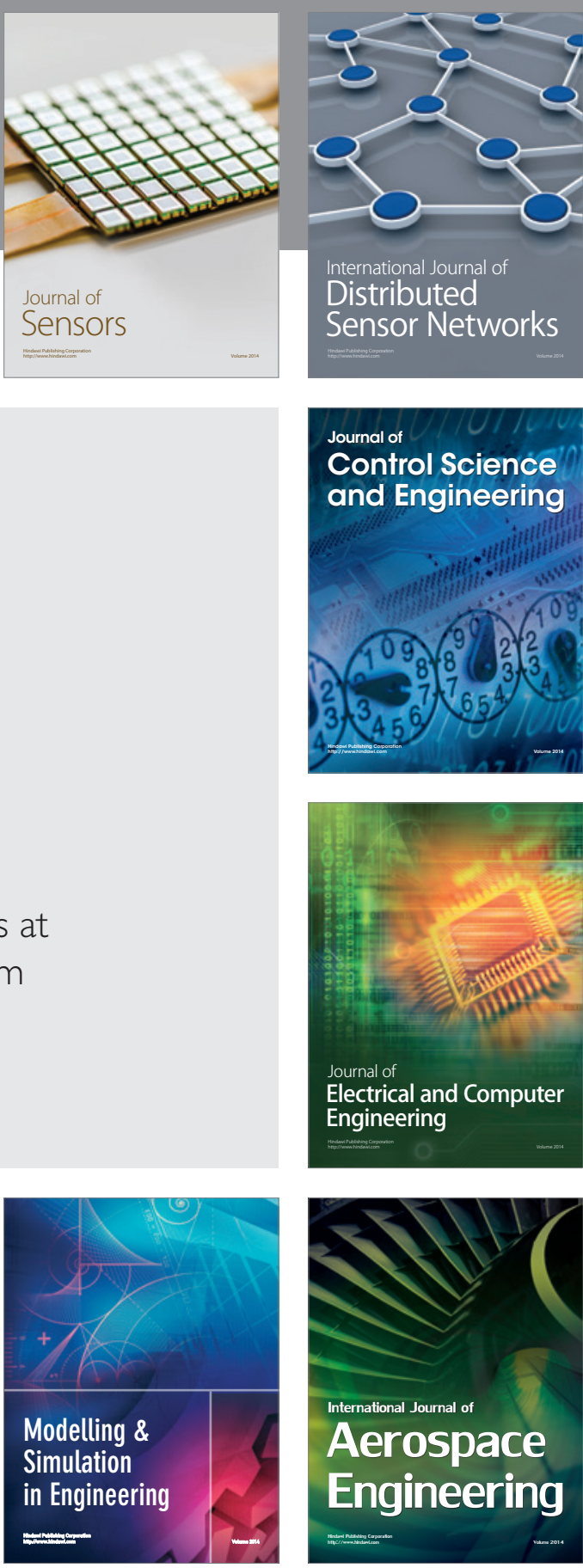

International Journal of

Distributed

Sensor Networks

Journal of

Control Science

and Engineering
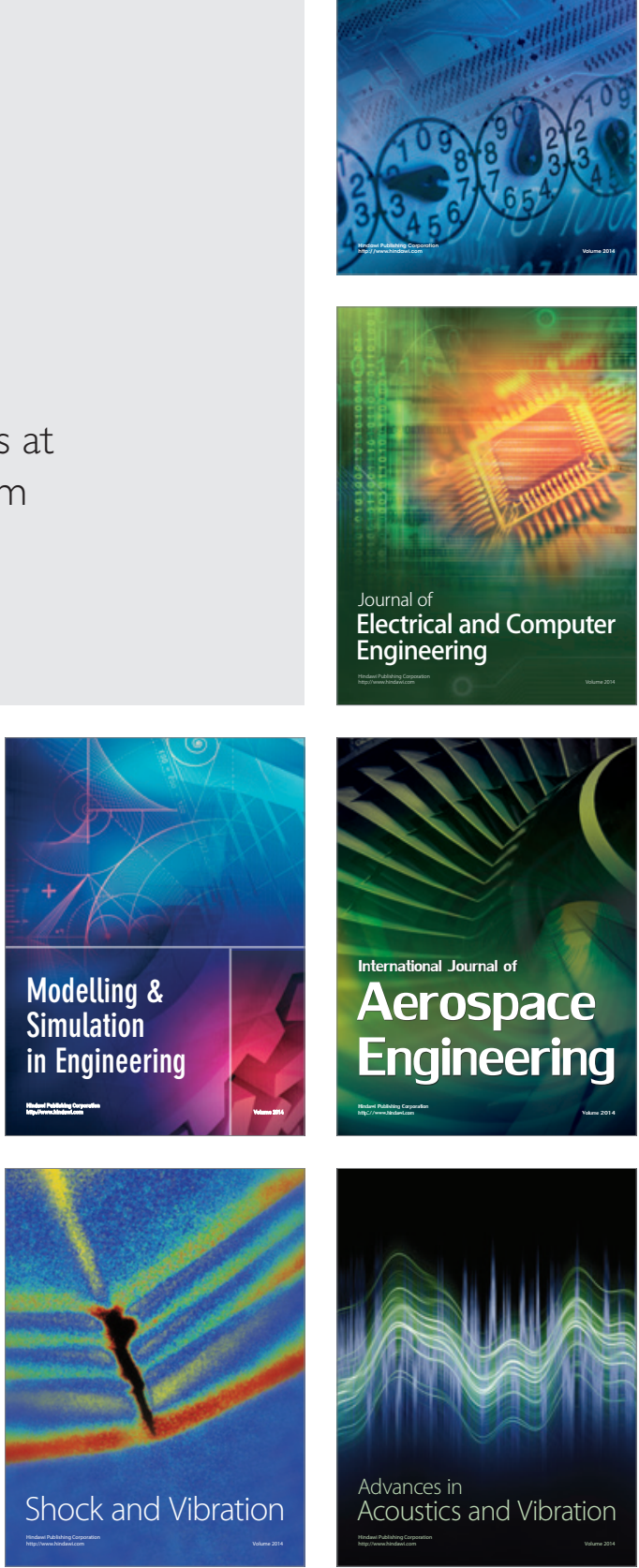